\title{
Estimation of Transient Response in a Pneumatic Pipeline with Turbulent Transition using a One-dimensional Model ${ }^{*}$
}

\author{
Mitsuhiro NAKAO ${ }^{* *}$, Kotaro KISHI $^{* *}$, Ryo KAWADA ${ }^{* * *}$, Kyotaro TANAKA $^{* * * *}$
}

State estimation of transient response flow in pneumatic pipelines is important for system design and phenomenon analysis. The purpose of this study is to investigate a one-dimensional numerical method by using a high-order finite upwind difference scheme that can calculate the transient response in a straight circular tube with turbulent transitions. In order to calculate such a flow by using a one-dimensional numerical model, the source term needs to be represented accurately. We propose an appropriate representation of the source term in the form of a switch between laminar and turbulent models with unsteady losses, depending on the critical Reynolds number. To validate the model, transient response experiments were conducted in a single closed-end circular tube with an inner diameter of $28 \mathrm{~mm}$ and a length of $4 \mathrm{~m}$, up to an initial pressure difference of $7 \mathrm{kPa}$, and the closed-end pressure was measured. Comparison with experimental data showed that the transient response flow including turbulent transition can be calculated accurately by considering the pipe wall roughness and setting the critical Reynolds number between 12000 and 14000 .

Keywords: Turbulent transition, Transient response, Simulation, Unsteady friction, One-dimensional model

\section{Introduction}

Estimation of state values such as pressure and flow rate in pneumatic pipelines is important for the design of pipeline systems and analysis of phenomena. The flow that occurs in pneumatic system is a mixture of laminar and turbulent flow and their transition regions, and the effect of compressibility may also have to be considered. Nakao and Kagawa ${ }^{1)}$ studied a one-dimensional model using the governing equations of a conservative system for the future purpose of developing a unified solution method for the flow in pneumatic pipelines and clarified its validity for small amplitude waves. In order to extend the method to a large amplitude regime, it is necessary to investigate the representation of the source term suitable for computations involving turbulent transition and compressibility. One of the examples of such flows which include this calculation is that of micro-pressure waves in tunnels ${ }^{2)}{ }^{3)}$. However, for micro-pressure waves in tunnels, only the compressional waves along the direction of the train travel needs to be considered, whereas in the case of flow in a

\footnotetext{
* $\quad$ Manuscript received June 11, 2021

** Graduate School of Science and Engineering, Kagoshima

University (Korimoto 1-21-40 Kagoshima, Japan)

E-mail: nakao@mech.kagoshima-u.ac.jp

*** Graduate School of Science and Engineering, Kagoshima

University (present affiliation is Azbil Corporation)

(Kawana 1-12-2 Fujisawa, Kanagawa, Japan)

***** Tokyo Gas Co. Ltd.

(Kaigan 1-5-20 Minato-ku,Tokyo, Japan)
}

pipeline, the reflections in the pipeline needs to be considered. Therefore, it is considered that the same method cannot be simply applied.

In this study, the transient response of air in a straight circular tube is used as an example including turbulent transition. In the one-dimensional numerical method using the high-order finite upwind difference method, the expression of the source term suitable for the calculation is investigate by comparison with experiments.

\section{Nomenclature}

$\begin{array}{lll}A & : & \text { coefficient matrix } \\ a, b & : & \text { correction factor of loss term } \\ c & : & \text { sound velocity } \\ c_{p} & : & \text { specific heat capacity at constant pressure } \\ D & : & \text { inner diameter of pipeline } \\ e & : & \text { total energy per unit volume } \\ f, g & : & \text { loss term } \\ H & : & \text { total enthalpy per unit mass } \\ p & : & \text { pressure } \\ P r & : & \text { Prandtl number } \\ Q & : & \text { variable vector } \\ R e & : & \text { Reynolds number }(\rho u D / \mu) \\ S & : & \text { source term matrix } \\ t & : & \text { time } \\ u & : & \text { velocity } \\ x & : & \text { coordinate along the pipeline }\end{array}$




$\begin{array}{lll}\gamma & : & \text { specific heat ratio } \\ \Delta P & : & \text { initial pressure difference } \\ \Delta t & : & \text { time increment } \\ \Delta x & : & \text { width of the computation grid } \\ \Delta \tau_{f} & : & \text { non-dimensional time increment } \\ & & \left(4 \mu \Delta t /\left(\rho D^{2}\right)\right) \\ \Delta \tau_{g} & : & \text { non-dimensional time increment } \\ \theta & : & \text { temperature } \\ \theta_{w} & : & \text { wall temperature } \\ \lambda & : & \text { friction factor } \\ \mu & : & \text { viscosity } \\ \rho & : & \text { density }\end{array}$

\section{Calculation method}

\subsection{Governing Equation}

Air flow in a pipe is three-dimensional, but if the crosssectional area is constant and the flow is axial, the flow can be treated as one-dimensional. When the one-dimensional Euler equation is locally linearized, the equations of continuity, motion, and energy with the source term added in the conservative form are expressed as follows ${ }^{1)}$ :

$$
\frac{\partial Q}{\partial t}+A \frac{\partial Q}{\partial t}+S=0
$$

where each matrix is defined as

$$
\begin{gathered}
A=\left(\begin{array}{ccc}
0 & 1 & 0 \\
-0.5(3-\gamma) u^{2} & (3-\gamma) u & \gamma-1 \\
-H u+0.5(\gamma-1) u^{3} & H-(\gamma-1) u^{2} & \gamma u
\end{array}\right), \\
Q=\left(\begin{array}{c}
\rho \\
\rho u \\
e
\end{array}\right), S=\left(\begin{array}{c}
0 \\
f(x, t) \\
g(x, t)
\end{array}\right)
\end{gathered}
$$

The loss terms $f(x, t)$ and $g(x, t)$ in the source term are the frictional loss term and heat transfer term, respectively, and can be calculated using the non-conservative loss terms $f^{*}(x, t)$ and $g^{*}(x, t)$ as follows:

$$
\begin{aligned}
& f(x, t)=\rho f^{*}(x, t) \\
& g(x, t)=\rho u f^{*}(x, t)+\frac{1}{\gamma-1} g^{*}(x, t)
\end{aligned}
$$

\subsection{Loss Terms in Laminar Flow}

The loss terms for the case of laminar flow in the nonconservative form are expressed by the following equations:

$$
\begin{aligned}
& f^{*}(x, t)=\frac{32 \mu}{\rho D^{2}}\left[u+\frac{1}{2} U(x, t)\right] \\
& g^{*}(x, t)=\frac{16(\gamma-1) \mu}{P r D^{2}} G(x, t)
\end{aligned}
$$

The convolution integrals $U(x, t)$ and $G(x, t)$ of equations (4) and (5) are expressed as follows

$$
\begin{aligned}
U(x, t) \approx & \sum_{i=1}^{n_{f}} y_{f_{i}}(x, t) \\
= & \sum_{i=1}^{n_{f}}\left\{e^{-n_{f_{i}} \Delta \tau_{f}} y_{f_{i}}(x, t-\Delta t)\right. \\
& \left.+m_{f_{i}} e^{-n_{f_{i}} \Delta \tau_{f} / 2}(u(x, t)-u(x, t-\Delta t))\right\} \\
G(x, t) \approx & \sum_{i=1}^{n_{g}} y_{g_{i}}(x, t) \\
= & \sum_{i=1}^{n_{g}}\left\{e^{-n_{g_{i}} \Delta \tau_{g}} y_{g_{i}}(x, t-\Delta t)\right. \\
& \left.+m_{g_{i}} e^{-n_{g_{i}} \Delta \tau_{g} / 2}(u(x, t)-u(x, t-\Delta t))\right\}
\end{aligned}
$$

where the coefficients $m_{f i}, n_{f i}, m_{g i}$, and $n_{g i}$ are the optimal values obtained by Nakao and Kagawa ${ }^{4)}$.

\subsection{Loss Terms in Turbulent Flow}

The non-conservative friction loss term in the case of turbulent flow is expressed in equation (8). The friction coefficient was evaluated using equation (9).

$$
\begin{aligned}
& f^{*}(x, t)=\frac{\lambda \rho u^{2}}{2 D}+\frac{16 \mu}{\rho D^{2}} U(x, t) \\
& \lambda=0.3164 R e^{-0.25}
\end{aligned}
$$

The second term on the right-hand side of equation (8) is the unsteady turbulence loss, which can be expressed by a convolution integral using the weighting function $W(\tau)$. This model is expressed by the following equation proposed by Vardy et al. ${ }^{5)}$.

$$
W(\tau)=0.25 f \operatorname{Re} \sum_{k=1}^{10} e^{-(k \pi f R e)^{2} \tau / 16}
$$

The heat transfer term is equation (11) for steady turbulent flow.

$$
g^{*}(x, t)=-4 h\left(\theta_{w}-\theta\right) /(\rho D)
$$

The heat transfer coefficient $h$ in the equation can be expressed using the Dittus-Boelter equation as follows:

$$
h=0.023 \operatorname{Re}^{0.8} \operatorname{Pr}^{0.4} k / D
$$

The thermal conductivity is approximated as

$$
k=4.8 \times 10^{-8}\left(\theta_{w}-273.15\right)+1.71 \times 10^{-5}
$$

As a heat transfer term in unsteady flow, Mashimo et al. adopt the following equation derived for flow induced by propagating shock waves, which is proposed by Hartunian et al.

$$
g^{*}(x, t)=\frac{4 c_{p}}{u D}\left(\theta_{w}-\theta\right) \operatorname{Pr}^{-2 / 3} f^{*}(x, t)
$$

\subsection{Treatment of Loss Terms}

In the analysis of micro-pressure waves in tunnels, the loss term differs depending on the conditions. Mashimo et al. observed a pressure increase of $1.2 \mathrm{kPa}$ near the entrance of a tunnel with an equivalent diameter of $9 \mathrm{~m}$. They used 
equations (8) and (14), assuming turbulent flow as the loss term. In contrast, the initial pressure increase in the compression wave treated by Aoki et al. is $6 \mathrm{kPa}$, but the inner diameter of the pipe is as narrow as $16 \mathrm{~mm}$. Equations (4) and (5) are used for the loss term, assuming laminar flow. In addition, the unsteady friction term parameter, which depends on the roughness and shape of the pipe wall, is considered, and the unsteady loss term is corrected as follows:

$$
f^{*}(x, t)=\frac{32 \mu}{\rho D^{2}} u+1.3 \times \frac{16 \mu}{\rho D^{2}} U(x, t)
$$

The heat transfer term is calculated using the equation obtained from the analysis of the unsteady laminar boundary layer behind the shock wave. However, since the heat transfer term includes the distance from the wavefront, it is not suitable for the analysis of pulsating flow in a pipe and is not treated in this study.

Based on the results of these previous studies, we can see that it is necessary to distinguish between turbulent and laminar flow depending on the calculation conditions. Therefore, we introduce a critical Reynolds number $\operatorname{Re}_{c}$ to switch between laminar and turbulent losses. It is known that the critical Reynolds number depends on the acceleration in pulsating flow, and it is considered that the turbulent transition does not occur at the same critical Reynolds number in transient flow as in steady flow.

As a fundamental investigation, it is assumed that the turbulent transition can be expressed by the Reynolds number defined by the same equation as the steady flow, and that the flow is laminar when the Reynolds number is less than a certain critical Reynolds number and turbulent when it is greater. In this study, the laminar flow loss, turbulence flow loss, and heat transfer term are combined and calculated, and the appropriate combination and the appropriate critical Reynolds number are investigated through comparison with experimental values.

\section{Method of Study}

A straight pipeline with an inner diameter of $28 \mathrm{~mm}$ and a length of $4 \mathrm{~m}$, shown in Fig.1, was used for the study. The inner diameter was chosen to be the value that could be used in actual piping. Since extending the pipeline with fittings may cause changes in the waveform, a $4 \mathrm{~m}$ pipeline, which is relatively easy to procure and can be prepared without fittings, was adopted. A differential pressure gauge (ALL SENSOR 5PSI-D-4V) was attached to the closed end, and the other end was opened to the atmosphere. The open-air side was closed with a rubber membrane, and the hand valve was opened to

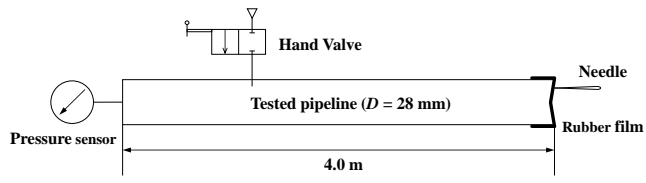

Fig.1 Experimental apparatus

\begin{tabular}{|c|c|c|}
\multicolumn{3}{|c}{ Table 1 Experimental conditions } \\
\hline$\Delta P$ & $\begin{array}{c}\text { Atmospheric } \\
\text { pressure } \mathrm{kPa}\end{array}$ & Temperature K \\
\hline $7 \mathrm{kPa}$ & 102.25 & 293.1 \\
\hline $5 \mathrm{kPa}$ & 102.06 & 296.4 \\
\hline $3 \mathrm{kPa}$ & 101.95 & 296.6 \\
\hline
\end{tabular}

allow air to flow into the pipe and pressurize it by $\Delta P$. After pressurization, the hand valve was closed, the rubber membrane was broken with a needle to generate a pressure pulsation, and the pressure change at the end of the pipe was measured with the differential pressure gauge. The initial pressure in the pipe was examined in three ways: $\Delta P=3 \mathrm{kPa}$, $5 \mathrm{kPa}$, and $7 \mathrm{kPa}$. Kitagawa et al. reported that in the transient response of a pneumatic pipeline with an initial pressure difference of $5 \mathrm{kPa}$, the calculated values of flow velocity and pressure assuming laminar flow did not agree with the experimental values due to the effect of turbulent transition ${ }^{6}$. It is also reported that the laminar flow calculation and experiment start to deviate from each other at the initial pressure difference of $2 \mathrm{kPa}$. In this study, in order to investigate the transient response including turbulent transition, the initial pressure difference was determined based on the paper by Kitagawa et $\mathrm{al}^{6}$.

The discretization method for the conservative governing equations, the third-order Chakravarthy-Osher scheme, which is relatively simple among the TVD (Total Variation Diminishing) high-resolution schemes, was used as in Nakao et $\mathrm{al}^{1)}$. As an initial condition for the calculations, the flow velocity was zero, and the atmospheric pressure and temperature were the values at the time of data acquisition shown in Table 1 . The viscosity coefficient was calculated using Sutherland's equation, which is expressed as

$$
\mu=1.81 \times 10^{-5}\left(\frac{\theta}{293.15}\right)^{1.5} \frac{404.15}{\theta+111}
$$

The width of the computation grids $\Delta x$ is set to $0.1253 \mathrm{~m}$, and the total number of computational grids is 33 . The time increment $\Delta t$ was determined by trial and error to be as large as possible within a stable range, and was set to $5.821 \times 10^{-5} \mathrm{~s}$. 


\section{Result and Discussions}

First, calculations assuming that the flow is entirely laminar are performed and compared with experimental data. In the conventional laminar flow model, the loss terms are calculated using equations (4) and (5), while equation (15) is used instead of equation (4) in the Aoki et al. model. Figure 2 shows the experimental and calculated results for an initial pressure of $\Delta P=7 \mathrm{kPa}$. In the conventional laminar flow model, the initial waveform shape of the transient response is close to the experimental value, but the decay of the waveform is underestimated. Aoki et al.'s model shows a slight improvement, but the tendency to underestimate the attenuation remains unchanged.

Next, the effect of the unsteady loss term in turbulent flow was investigated in a model assuming turbulent transition. The critical Reynolds number was set to 4000, and when the Reynolds number was less than 4000, calculations were performed using the normal laminar flow model. When the Reynolds number is greater than that, the flow is considered turbulent. Two types of turbulence models are considered: steady turbulence using the first term on the right-hand side of equation (8) and equation (11), and unsteady turbulence using equation (8) and equation (14). Figure 3 shows the experimental and calculated results for the initial pressure $\Delta P$ $=7 \mathrm{kPa}$. The initial waveform of the transient response is in good agreement with the results of both calculations. However, the shape of the waveform after the passage of time is also close to a rectangle, which is different from the experimental shape. The calculated shape of the unsteady term is somewhat closer to the experimental value, indicating that the calculation of the unsteady term in turbulent flow is necessary. It can also be seen that the calculated waveform attenuation is smaller than the experimental one, even if the turbulent transition is simply taken into account. Therefore, we attempted to improve the attenuation by extending the method of Aoki et al.

In turbulent flow, the friction factor varies with the surface roughness. Since the above calculation assumes a smooth pipeline, the coefficients may be different from the actual values. Therefore, we developed a correction equation (17) that takes into account the roughness of the pipe wall.

$$
f^{*}(x, t)=a \frac{\lambda \rho u^{2}}{2 D}+b \frac{16 \mu}{\rho D^{2}} U(x, t)
$$

Here, $a$ and $b$ are the correction factors. This equation is in the form of applying the idea of Aoki et al. to the turbulent regime

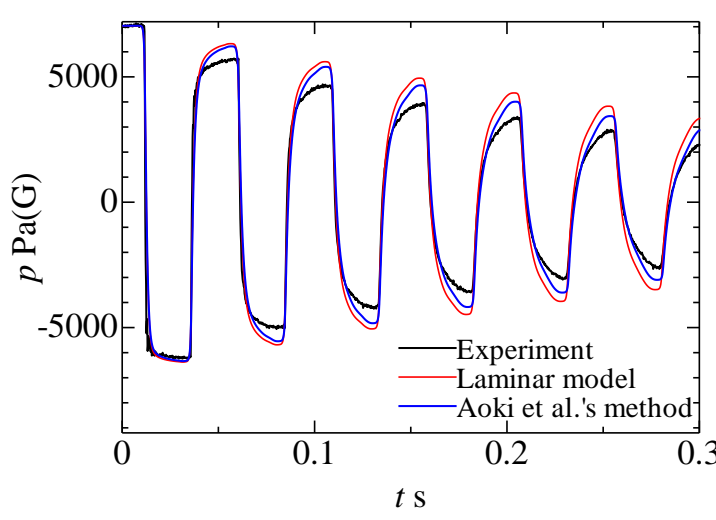

Fig.2 Comparison of experimental values, the ordinary laminar flow model and Aoki et al.'s method at $\Delta P=7 \mathrm{kPa}$

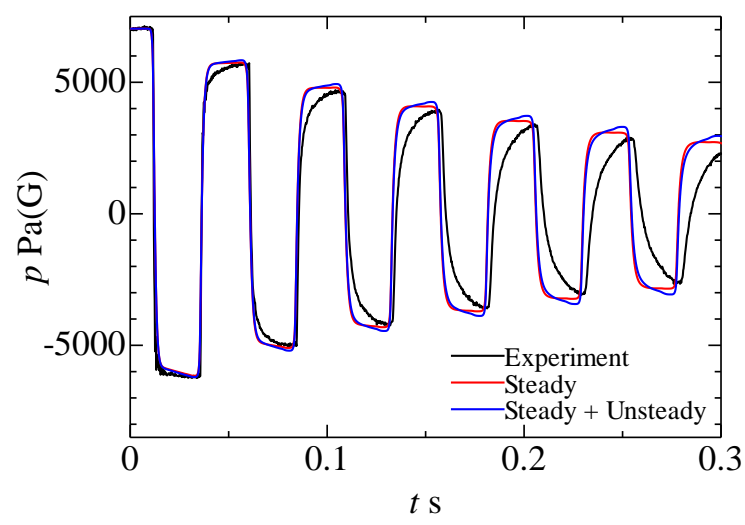

Fig.3 Comparison of experimental data with calculations considering transition to steady turbulence (Steady) and calculations considering transition to unsteady turbulence (Steady + Unsteady) at $\Delta P=7 \mathrm{kPa}$ and $R e_{c}=4000$

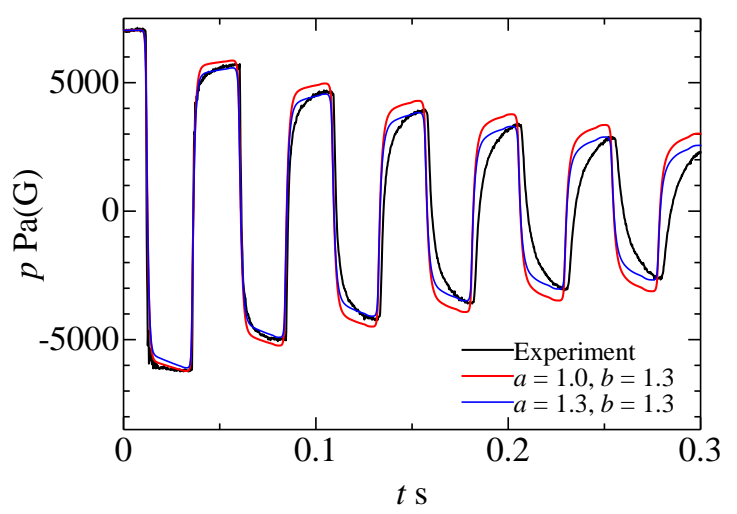

Fig.4 Comparison between the experimental data and the calculation using equation (17) at $\Delta P=7 \mathrm{kPa}$ and $R e_{c}=4000$

and also considering the correction for the steady-state term. The value of the correction factor a, obtained from the Moody diagram, is 1.3 when the roughness of the pipe wall is about 


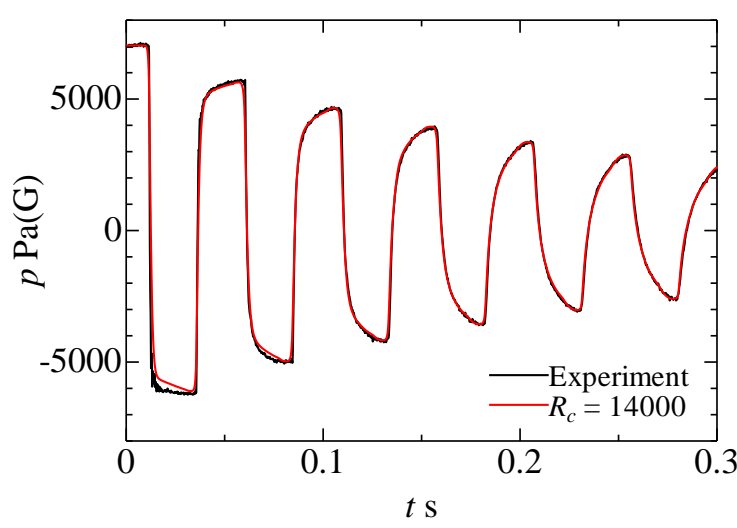

Fig.5 Comparison between the experimental data and the calculation at $\Delta P=7 \mathrm{kPa}$ and $R e_{c}=14000$

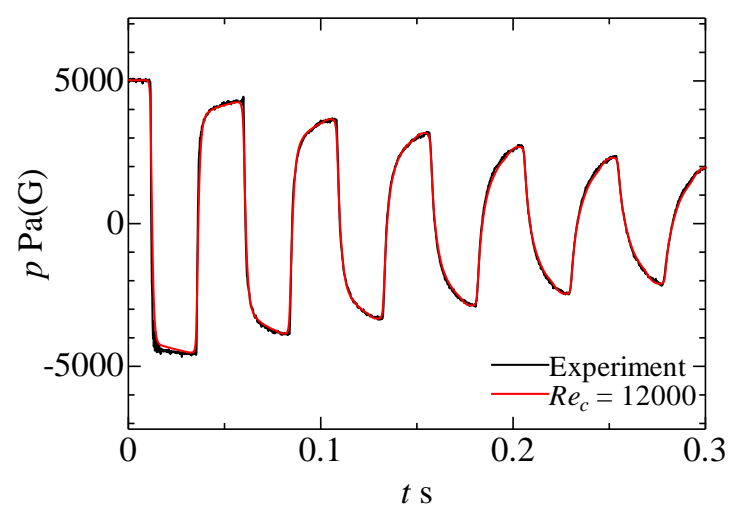

Fig.6 Comparison between the experimental data and the calculation at $\Delta P=5 \mathrm{kPa}$ and $R e_{c}=12000$

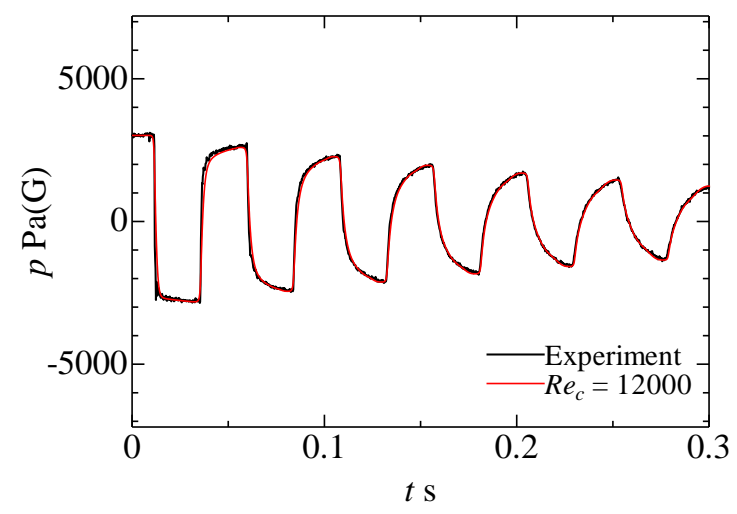

Fig.7 Comparison between the experimental data and the calculation at $\Delta P=3 \mathrm{kPa}$ and $R e_{c}=12000$

$0.1 \mathrm{~mm}$. Figure 4 compares the results of the calculation with $a=1.0, b=1.3$ and the calculation with $a=1.3, b=1.3$ with the experimental data for the calculation with turbulent transition $\left(\operatorname{Re}_{c}=4000\right)$. Note that the correction for the steady term was applied only for turbulent flow, while the correction for the unsteady term was applied for both laminar and turbulent flows. From the figure, it can be seen that the results for $a=1.3$ and $b=1.3$ are in good agreement with the experimental values in terms of amplitude. However, the approximate shape of the waveform does not match.

Therefore, the value of the critical Reynolds number was varied from the value in the steady state flow, and the appropriate value was obtained by trial and error by comparing the pressure calculation results with the experimental data. The results of calculations using the obtained critical Reynolds number are shown in Figure 5, Figure 6, and Figure 7 for the initial pressure difference of $\Delta P$ $=7 \mathrm{kPa} 5 \mathrm{kPa}$, and $3 \mathrm{kPa}$, respectively. In all cases, the calculated and experimental values are in excellent agreement. For the initial pressure difference up to $7 \mathrm{kPa}$ in this experiment, the appropriate value was found to be around 12,000 to 14,000 .

It is known that the critical Reynolds number in pulsating flow is a function of dimensionless frequency and differs from its value in steady flow ${ }^{7}$. However, since the transient flow contains multiple frequency components and is not a simple single-frequency pulsating flow, it is not known how the critical Reynolds number should be described. In this study, we investigate the possibility of capturing turbulent transitions in transient response flows by setting the critical Reynolds number to an appropriate value. Identifying an appropriate way to describe the critical Reynolds number is our future task.

\section{Conclusions}

In the present study, the transient response of air in a straight circular tube with turbulent transition is calculated using a high-order upwind finite difference method. In the transient response up to an initial pressure difference of $7 \mathrm{kPa}$, it was confirmed that the experimental and computational results agreed well by switching between laminar and turbulent flows using the critical Reynolds number, considering the pipe wall roughness, and adding a correction factor to the loss term.

\section{References}

1) Nakao, M., Kawashima, K., Kagawa, T.: High Speed and Accurate Computing Method for Transient Response of Pneumatic Transmission Line Using Conservative Governing Equations, Transactions of the Japan Fluid Power System Society, Vol.44, No.2, p.35-42 (2013) 
2) Mashimo, S., Nakatsu, E., Aoki, T., Matsuo, K.: Attenuation and Distortion of a Compression Wave Propagating in a High-Speed Railway Tunnel, Transactions of the Japan Society of Mechanical Engineers. B., Vol.62, No.597, p.1847-1854 (1996)

3) Aoki, T., Ishii, S., Sasa, D.: Effects of Initial Waveform on the Nonlinear Propagation of Compression Wave in a Tunnel, JSME Annual Meeting, Vol.2, p.155-156 (2009)

4) Nakao, M., Kagawa, T.: Numerical Error in Method of Characteristics with Weighting Function-based Model for Small Amplitude Wave in Gas Pipeline, Transactions of the Japan Fluid Power System Society, Vol.46, No.4, p.23-31 (2015)
5) Vardy, A. E., and Brown, J. M. B.: Transient Turbulent Friction in Smooth Pipe Flows, Journal of Sound and Vibration, Vol.259, No.5, p.1011-1036 (2003)

6) Kitagawa, A., Kagawa, T., Sanada, K., Okada, T.: A Study on the Transient Characteristics of the Flow in Pneumatic Transmission Line, Transactions of the Society of Instrument and Control Engineers, Vol.33, No.4, p.227-233 (1997)

7) Çarpinlioğlu, M. Ö, Gündoğdu, M. Y.: A Critical Review on Pulsatile Pipe Flow Studies Directing Towards Future Research Topics, Flow Measurement and Instrumentation, Vol.12, p.163-174 (2001) 